\title{
Propuesta de integración, en soporte hipermedia, de la educación ambiental en el aula de matemáticas.
}

\author{
Juan Carlos Pereña Moro \\ a11550@usal.es \\ (C. P. "Villa de Felipe II", Villoruela - \\ Salamanca-) \\ Ángela Barrón Ruiz \\ ansalusal.es \\ José $M^{a}$ Chamoso Sánchez \\ jchamosolusal.es \\ Universidad de Salamanca
}

吅虽

\section{1.- Introducción}

De acuerdo con CORIAT (1997) entendemos que no es posible tener conciencia medioambiental sin referencias a las Matemáticas. Por otro lado, no se puede olvidar que la enseñanza transversal de la Educación Ambiental es una prioridad educativa, así como una enseñanza obligatoria, para todos los niveles de la Educación Obligatoria en España (LOGSE, 1990). Además, es preciso actualizarse y utilizar recursos novedosos que puedan contribuir a la mejora del proceso de enseñanza-aprendizaje. Por esto, el contenido se pretende presentar con una herramienta hipermedia. Estas tres realidades articulan el trabajo en tres bloques:

- Educación Ambiental: Dada la creciente preocupación social sobre el deterioro del medio ambiente natural y social, se pretende incrementar la sensibilización del alumnado hacia la necesidad de mantener una relación más preservadora de los equilibrios ecológicos, contribuyendo con ello a una formación más integral de la persona.

- Matemáticas: Su relevancia para la formación general de la persona, así como su elevado fracaso en el alumnado, obliga a la búsqueda de metodologías más novedosas para su estudio; por otra parte, las Matemáticas se consideran como las más adecuadas para desarrollar un proyecto en este sentido, pues, como plantea STEWART, están presentes "en cualquier nivel de la vida; sólo hay que mirar para verlo" (1991, 41); no en vano dicho autor se refirió a ellas como el segundo secreto de la vida después del descubrimiento de la estructura molecular del ADN (ácido desoxirribonucleico).

Hipermedia: Las nuevas tecnologías de la información y la comunicación han permitido el descubrimiento de nuevos recursos didácticos con interesantes posibilidades educativas. Además, el escaso número de trabajos en soporte hipermedia que articulen la enseñanza de las Matemáticas con la Educación Ambiental anima a realizar el trabajo en este sentido.

\section{2.- Objetivos}

Con la base en el planteamiento anterior, se pueden sintetizar los objetivos de esta propuesta en los siguientes: 
Sensibilizar al alumnado en cuestiones medioambientales y promover una relación más preservadora de los equilibrios ecológicos.

Aumentar la motivación de los alumnos hacia las Matemáticas al integrar contenidos y problemáticas de su realidad socioambiental.

Favorecer una mayor autonomía en la adquisición de los aprendizajes al potenciar la actividad autorreguladora del alumno en la interacción con la herramienta hipermedia diseñada.

- Incrementar la significatividad de los aprendizajes.

\section{3.- Planteamientos teóricos}

Para el diseño de la herramienta se han utilizado trabajos previos (CHAMOSO, HERNÁNDEZ, LÓPEZ, MARTÍN, y RODRÍGUEZ, 2002) en los que se había abordado la integración de un soporte hipermedia en la enseñanza de las Matemáticas. Ahora, además, se pretende su adaptación en función de los planteamientos de una pedagogía constructivista (BARRÓN RUIZ, 1991).

Unas de las formas para conseguir que el conocimiento sea más significativo para el alumno es que parta de su propia experiencia y sus conocimientos, y teniendo en cuenta sus intereses, sus capacidades y sus concepciones a lo largo de todo el proceso. Por ello se quiere utilizar el propio ambiente circundante.

Por otro lado, los problemas y las actividades no deben presentarse como una sucesión progresiva basada, únicamente, en la lógica disciplinar. Su desarrollo también ha de tener en cuenta la psicología del proceso evolutivo. Una manera de considerar este criterio es por medio de herramientas que permitan crear el propio conocimiento y avanzar en el mismo de la forma en que parezca más adecuado, sabiendo siempre en el lugar en que uno se encuentra... Además, en el marco de las nuevas teorías de aprendizaje, se pretende que el profesor no sea un mero transmisor de conocimientos sino que sea un orientador del estudiante.

Los sistemas hipermedia permiten abordar la enseñanza de una forma diferente a la que se hace con otros materiales educativos pudiendo aportar mejoras en la educación: modelos no lineales, integración de texto, sonido, locución, imagen, animación, vídeo, interacción y enlaces en un mismo soporte.

Estas características, al ser presentadas de forma conjunta, incrementan sus posibilidades independientes como que el estudiante pueda acceder a la información a su propio ritmo, permitiendo su propia autonomía (lo que está en el marco de las normativas educativas actuales, LOGSE, 1991; LOCE, 2002). Además, su formato abierto y flexible le permite desarrollar actividades en espiral con ramificaciones que pueden adaptarse a cada usuario.

Por otra lado, la motivación inicial del alumnado puede verse incrementada con el empleo de un soporte hipermedia que permita una presentación atractiva en la que aparecen colores, sonidos, figuras, representaciones, etc., aspectos importantes para cualquier tipo de presentación. Ello posibilita un acercamiento a situaciones similares de la vida real (PADILLA DOMÍNGUEZ, MORINES BURGOS, RODRÍGUEZ CELOS, SÁNCHEZ MERINO, 2000).

Otra importante justificación para el desarrollo educativo de recursos hipermedia son sus posibilidades en la organización del conocimiento. El conocimiento matemático no es lineal sino que está organizado en forma de redes proposicionales cuyos nodos se conectan entre sí por múltiples enlaces transversales y de distinto nivel, lo que hace que sea difícil plasmarlo en la estructura lineal del libro de texto. En cambio, los sistemas hipermedia permiten estructurar la información en bloques, conectados mediante enlaces, a través de los cuales se puede recuperar la información almacenada cuando sea necesario. Al abordar un determinado conocimiento se hace constante alusión a conceptos 
conocidos o tratados con anterioridad. Frente a la organización lineal del libro de texto, la estructura reticular de los sistemas hipermedia permite establecer enlaces que emulan la organización del conocimiento. A través de ellos se puede acceder de unas partes a otras de forma sencilla, consultar el significado de aquellas palabras que se desconocen, acceder a distintos tipos de ayuda o a elementos que pueden ser de utilidad (enlazar con una página web, utilizar programas de gráficos, usar una calculadora, etc.). Trabajar los contenidos de esta forma, similar a la organización de la mente humana, permite que el usuario vincule el nuevo conocimiento con el existente, favoreciéndose así un aprendizaje más significativo (LÓPEZ, 1999).

Las herramientas que se utilicen para representar el conocimiento tienen un impacto considerable en la adquisición del mismo por parte de los sujetos. En este sentido, cuando se muestra un conocimiento con representaciones diversas (imágenes, sonidos, simulaciones, animaciones...) se favorece su interiorización. A ello hay que añadir la simulación, pues los dibujos o representaciones en movimiento facilitan la comprensión de procesos dinámicos.

Por otro lado, la interactividad, unida a la retroalimentación, permite adaptar el ritmo y nivel de trabajo de cada usuario al control activo que éste pueda o quiera ejercer. Es decir, posibilita establecer una relación entre la persona que ejecuta una determinada actuación y la máquina que ofrece una respuesta ante la misma. Por ejemplo, puede corroborar que el camino elegido es el adecuado, ofrecer ayudas para solucionar dificultades, remarcar errores, sugerir caminos alternativos... Con ello, el usuario tiene la posibilidad de insistir en aquellos aspectos que le presenten dificultades, recordar o aprender conceptos que no tenga suficientemente asimilados o avanzar en aquellos puntos que ya domine con seguridad. Además, puede realizar una evaluación que permita al alumno y al docente obtener información para controlar el desarrollo del proceso de enseñanza-aprendizaje (CATALÁN y CATANY, 1996).

Con este planteamiento, el profesor debe modificar su cometido usual, pues los alumnos tienen responsabilidad en su propio aprendizaje y evaluación: construyen su propio conocimiento de forma más acusada que con otros soportes, bajo la dirección de un docente pero sin la necesidad de la presencia constante de éste. Por tanto, la labor del profesor es crear un nuevo contexto de enseñanza, desarrollar actividades variadas, redefinir estrategias, cambiar la manera de presentación de la información matemática, organizar la evaluación, etc. No se trata de que realice el diseño e implementación de las actividades hipermedia por sí mismo, pero sí que las organice según su concepción de enseñanza.

\section{Diseño de la herramienta}

Los destinatarios de esta herramienta debían ser aquellos alumnos que, por sus características psicoevolutivas, ven ampliada su capacidad de estudio autónomo, análisis de alternativas y toma de decisiones, y tienen mejor disposición para el trabajo cooperativo. Eso se corresponde con estudiantes de 12 a 14 años (primer ciclo de E.S.O.).

Para la presentación de los contenidos hubo que tener en cuenta que:

- Las informaciones tenían que aparecer enlazadas de forma no lineal.

- La organización tenía que desarrollarse en torno a núcleos de interés.

- La presentación y el diseño de pantallas debería cuidar la forma de la letra (adecuada en tamaño y suficiente en contenido), sintaxis de las frases (comprensible y correcta) e interfaz (navegación sencilla).

- Se incluyan ambientes para la exploración que fomenten la actividad autorreguladora y estructurante del alumno, con el fin de potenciar su investigación y descubrimiento (Barrón, 1997). 
Con esa premisa se organizaron los contenidos que se hicieron en torno a seis temas cotidianos de la vida de cualquier ciudadano: agua, residuos sólidos urbanos, energía y electricidad, ahorro y reciclado de papel, reparto de recursos, emisiones de $\mathrm{CO}_{2}$. Como herramienta de autor se utilizó Macromedia Director y su lenguaje de programación asociado Lingo.

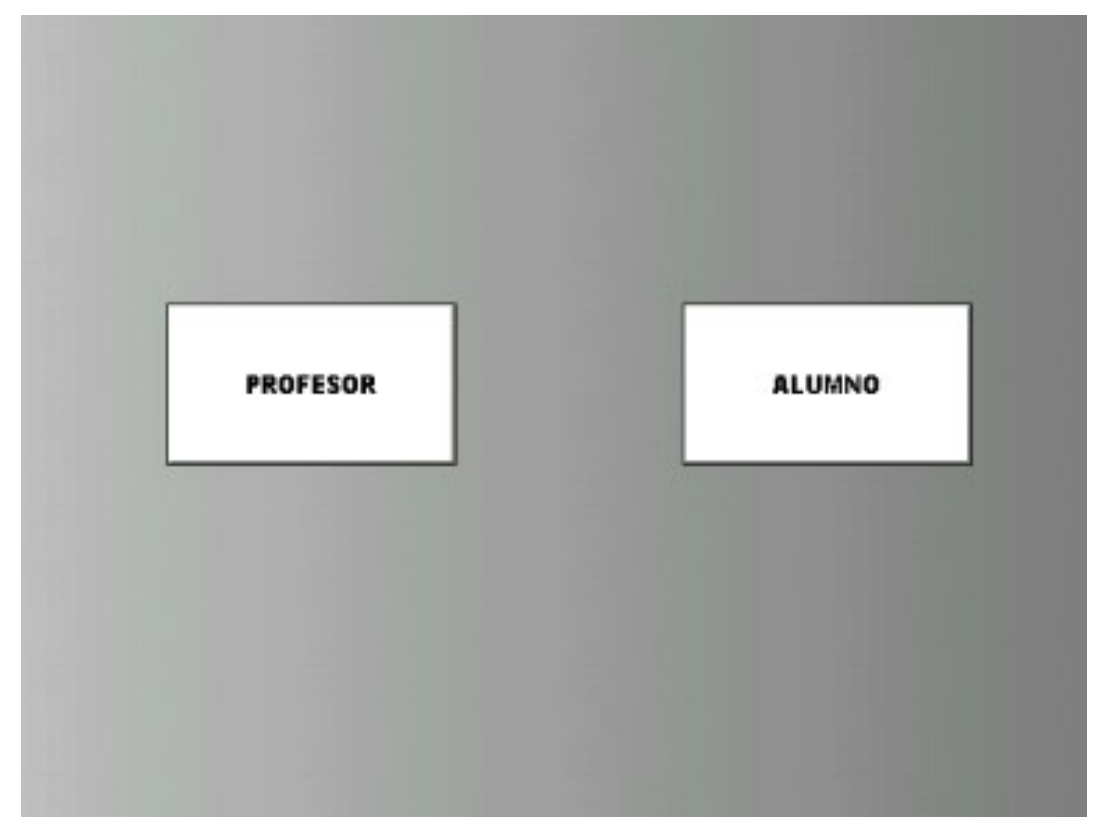

Figura 1. Pantalla inicial

Al instrumento se accedería mediante una pantalla inicial que permite dos modalidades: profesor o alumno (ver Figura 1). Nos centramos en la segunda de ellas por ser la que se dirige al estudiante. Una vez que se accede al icono "Alumno", aparece una pantalla de menú que permite elegir cualquiera de los temas en torno a los que se puede trabajar (Figura 2), en cada uno de los cuales se presentan otros subtemas (Figura 3). El menú es distinto para cada uno de ellos pero la concepción es general: desde la situación mundial hasta la local, pasando por los distintos niveles intermedios. Además, en cada pantalla aparecen otras opciones:

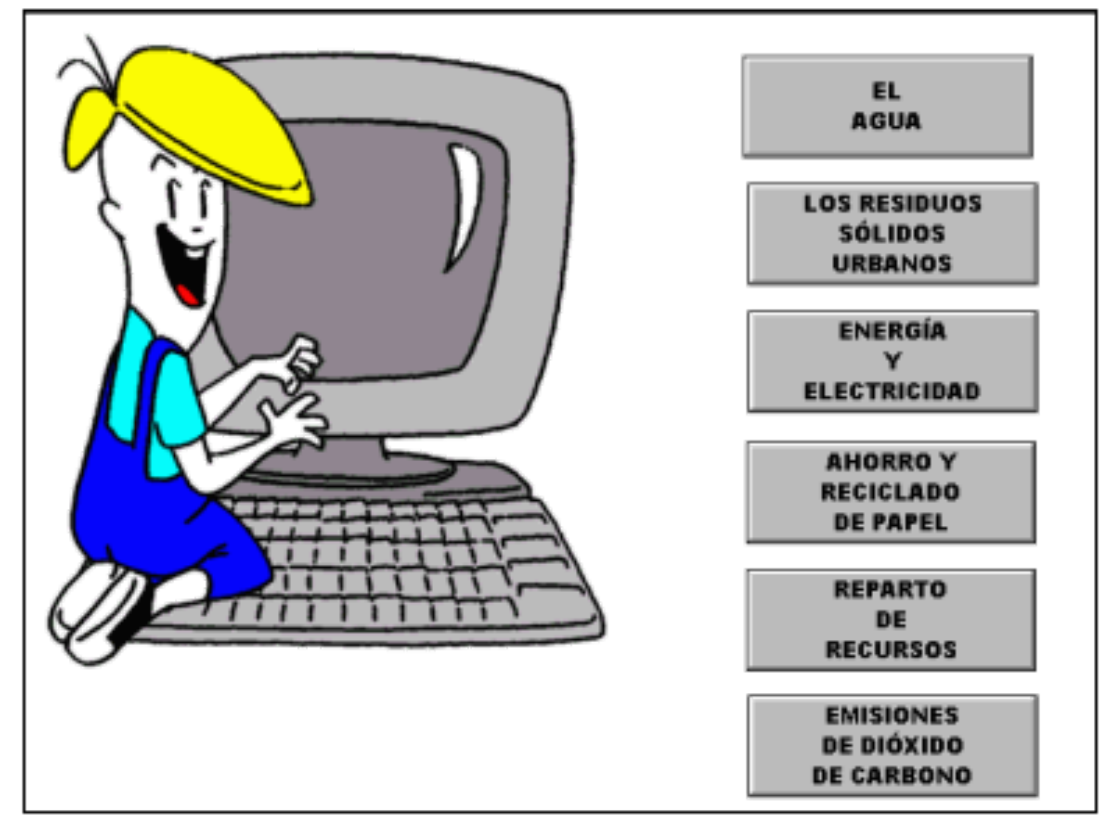

Figura 2. Pantalla de menú de temas

Contextualización y sensibilización: Su objetivo es conectar con los conocimientos previos del alumno y sensibilizarlo sobre el tema seleccionado. 
Búsqueda de soluciones: A la vista de lo tratado, se trata de poner los medios necesarios para que el estudiante saque las conclusiones pertinentes para poder contribuir a la protección del medio ambiente en su entorno inmediato y en su vida diaria.

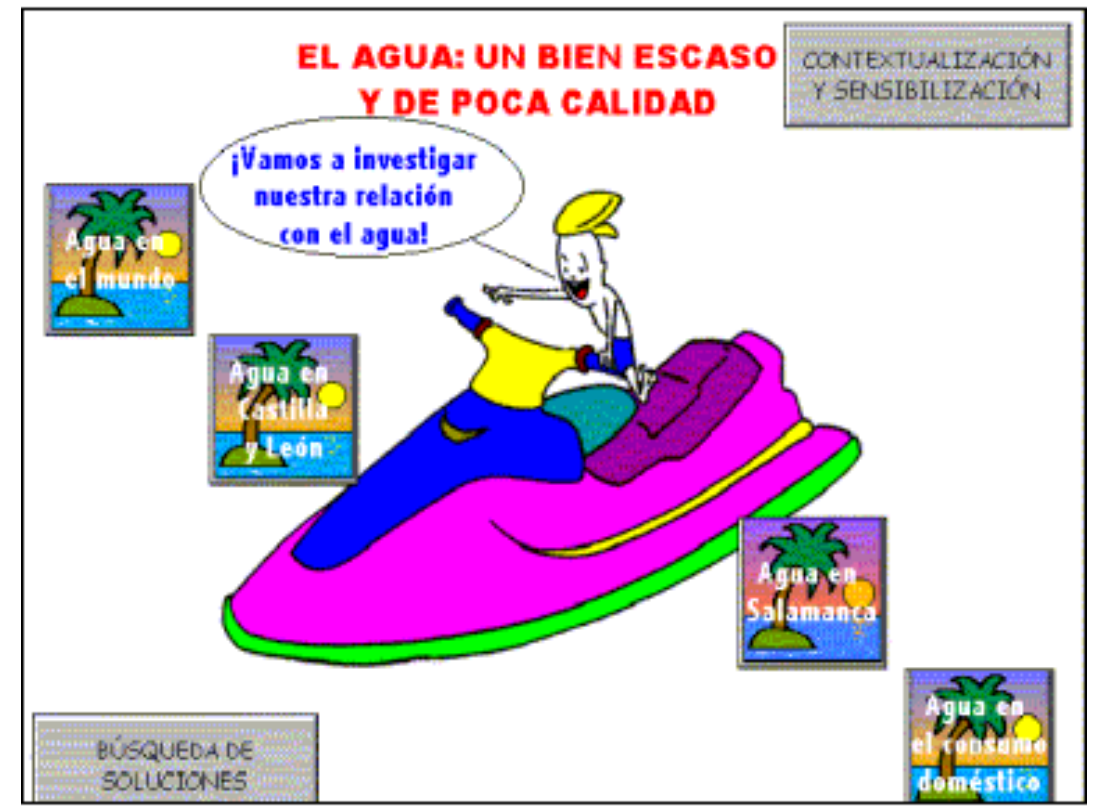

Figura 3. Pantalla de menú de subtemas

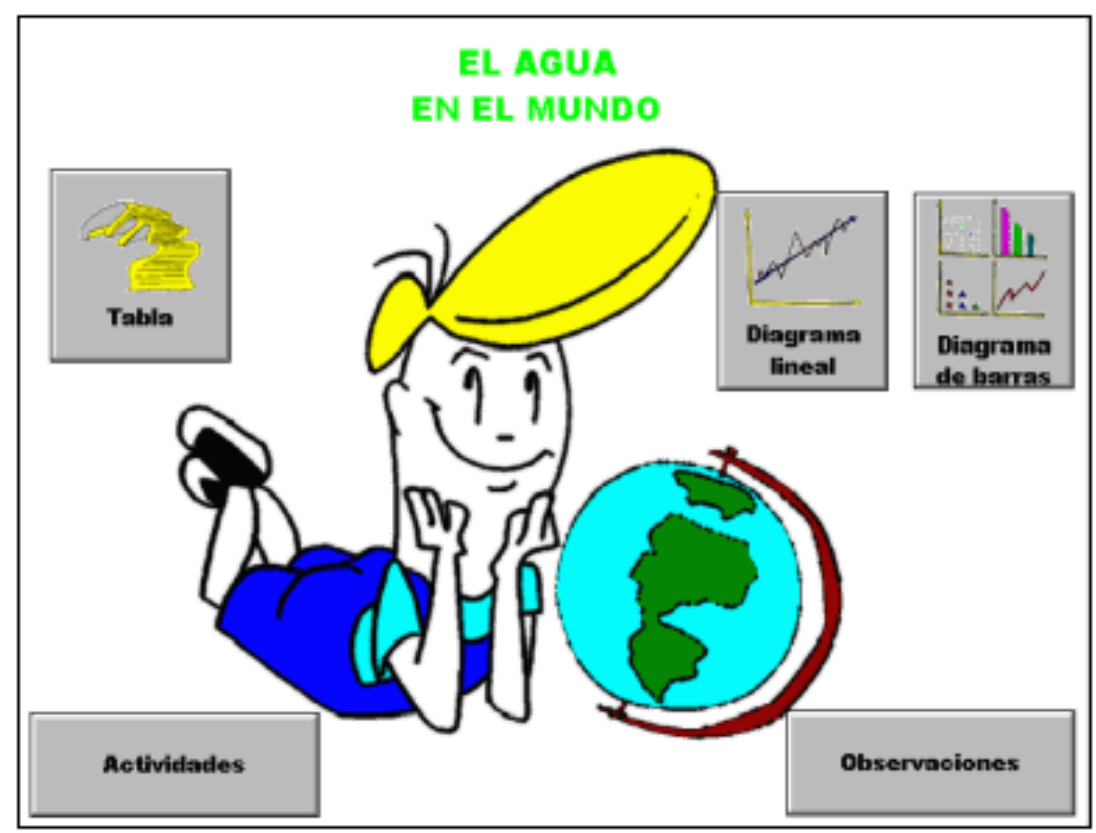

Figura 4. Pantalla de menú de cada subtema

Un paso posterior permite acceder, activando cada subtema, a las diversas actividades que se presentan con las siguientes opciones (ver, por ejemplo, Figura 4):

Tabla: En ella se recogen datos matemáticos reales a partir de los cuales se desarrollan las actividades.

Diagramas: Los datos anteriores se acompañan de una imagen que ilustre su contenido.

Actividades: Plantean una situación problemática.

Observaciones: Ofrecen datos complementarios que facilitarán la comprensión y concienciación del alumno sobre la problemática ambiental abordada. 
Esta propuesta se pretende evaluar con un diseño pretest-postest, para lo cual, habría que tener en cuenta los siguientes elementos:

Rendimiento y motivación hacia las Matemáticas y sensibilización medioambiental por medio de una prueba objetiva con ítems de opción múltiple, un cuestionario de preguntas abiertas y una escala de actitudes tipo Likert.

Calidad del programa, donde se pretende apreciar si incrementa la significatividad de los aprendizajes e intensifica la conducta autorregulada de los alumnos. Como instrumento, se adaptaría un protocolo de evaluación de materiales multimedia (por ejemplo, MARQUÈS, 1999).

\section{Conclusiones}

Con el diseño de esta herramienta se ha pretendido integrar dos realidades en un mismo recurso, la enseñanza y la educación, aunque no se puede olvidar que la segunda es mucho más amplia que la primera. Para ello se utiliza un recurso novedoso adaptado a las nuevas tecnologías de la información que pretende otorgar un mayor protagonismo al alumno y reforzar la labor docente.

Enseñar matemáticas es una labor compleja que se puede ver acrecentada si se pretende educar en valores medioambientales. Pero ello es un reto, además de ser una de las recomendaciones oficiales del curriculum español de la enseñanza obligatoria.

\section{7.- Referencias}

BARRÓN RUIZ, A. (1991) Constructivismo y desarrollo de aprendizajes significativos, Revista de Educación 294, 301-321

BARRÓN RUIZ, A. (1997) Aprendizaje por descubrimiento. Análisis crítico y reconstrucción teórica. Salamanca, Amarú. $2^{\mathrm{a}}$ ed.

CATALÁN, A. y CATANY, M. (1996) Educación Ambiental en la Enseñanza Secundaria. Madrid: Miraguano.

CORIAT, M. (1997) Cultura, educación matemática y currículo, en L. RICO (ed.) Bases teóricas del currículo de Matemáticas en Educación Secundaria, 151-209. Madrid, Síntesis.

CHAMOSO, J. M.; HERNÁNDEZ, L.; LÓPEZ, R.; MARTÍN, J. y RODRÍGUEZ, M. (2002) La simulación de un ábaco interactivo para la comprensión de la numeración elemental. Actas del V Simposio sobre aportaciones del área de Didáctica de las Matemáticas a diferentes perfiles profesionales, pp. 167-174. Universidad de Alicante, febrero 2002.

Ley Orgánica 1/1990, de 3 de octubre, de Ordenación General del Sistema Educativo.

Ley Orgánica 10/2002, de 23 de diciembre, de la Calidad de la Enseñanza.

LÓPEZ, R. (1999) Desarrollos curriculares de la ciencia de computadores en la enseñanza elemental. Tesis doctoral. Salamanca, Universidad de Salamanca.

MARQUÈS, P. (1999) Evaluación de materiales multimedia. Documento electrónico: <URL: http://www.xtec.es/ p pmarques/edusoft.htm>.

PADILLA DOMÍNGUEZ, Y.; MORINES BURGOS, J. F.; RODRÍGUEZ CELOS, P.; SÁNCHEZ MERINO, S. (2000) El ordenador. Una herramienta útil en la enseñanza-aprendizaje de las Matemáticas. Actas del IX Congreso sobre enseñanza y aprendizaje de las Matemáticas "Thales", 59- 
61, Cádiz.

STEWART, I. (1999), El segundo secreto de la vida. Barcelona: Crítica.

(C) Ediciones Universidad de Salamanca 